\title{
Delayed Cerebral Toxoplasmosis in a Kidney Transplant Patient: a Case Report
}

\author{
Hosung Myeong ${ }^{1}$, , Moowan Park', Ji Eun Kim³, Sun Won Park', Sang Hyung Lee ${ }^{1,2, *}$ (]) \\ 'Department of Neurosurgery, Seoul Metropolitan Government-Seoul National University Boramae Medical Center, Seoul 07061, Korea; \\ ${ }^{2}$ Department of Neurosurgery, Seoul Metropolitan Government-Seoul National University Boramae Medical Center, Seoul National University College \\ of Medicine, Seoul 07061, Korea; 'Department of Pathology, Seoul Metropolitan Government-Seoul National University Boramae Medical Center, \\ Seoul National University College of Medicine, Seoul 07061, Korea; ${ }^{4}$ Department of Radiology, Seoul Metropolitan Government-Seoul National \\ University Boramae Medical Center, Seoul National University College of Medicine, Seoul 07061, Korea
}

\begin{abstract}
Cerebral toxoplasmosis is often life-threatening in an immunocompromised patient due to delayed diagnosis and treatment. Several differential diagnoses could be possible only with preoperative brain images of cerebral toxoplasmosis which show multiple rim-enhancing lesions. Due to the rarity of cerebral toxoplasmosis cases in Korea, the diagnosis and treatment are often delayed. This paper concerns a male patient whose cerebral toxoplasmosis was activated 21 years post kidney transplantation. Brain open biopsy was decided to make an exact diagnosis. Cerebral toxoplasmosis was confirmed by immunohistochemistry and PCR analyses of the tissue samples. Although cerebral toxoplasmosis was under control with medication, the patient did not recover clinically and died due to sepsis and recurrent gastrointestinal bleeding.
\end{abstract}

Key words: Cerebral toxoplasmosis, kidney transplantation, multiple rim-enhancing lesion, PCR, brain open biopsy

\section{INTRODUCTION}

Toxoplasmosis is a relatively common infection caused by the intracellular parasite Toxoplasma gondii. More than onethird of the world's human population is known to be infected [1]. This parasite invades in a subclinical manner, and clinical problems are generally not manifested in immunocompetent individuals. Nevertheless, immunocompromised individuals may be prone to reactivation of cerebral toxoplasmosis, who present with headache, fever, seizures, focal neurologic deficits, or mental changes [2].

The original route of transmission is known to be ingestion of contaminated food or water with feces of infected cats [3]. In solid organ transplant patients, however, toxoplasmosis can be transmitted via seropositive organ donors or can be the reactivation of latent disease due to the use of immunosuppressants [4].

Owing to the maximal use of immunosuppressants in the

- Received 14 October 2021, revised 7 December 2021, accepted 21 December 2021. *Corresponding author (nslee@snu.ac.kr)

(c) 2022. Korean Society for Parasitology and Tropical Medicine

This is an Open Access article distributed under the terms of the Creative Commons Attribution Non-Commercial License (https://creativecommons.org/licenses/by-nc/4.0) which permits unrestricted non-commercial use, distribution, and reproduction in any

medium, provided the original work is properly cited. acute phase after transplantation, most toxoplasmosis occurs within 3 months [5]. Late presentation of toxoplasmosis after kidney transplantation is rare. Here, we report a patient whose cerebral toxoplasmosis was confirmed via brain open biopsy 21 years after kidney transplantation.

\section{CASE DESCRIPTION}

A 57-year-old male who had been experiencing gait disturbance, hallucinations, and cognitive impairment for approximately 10 days was brought into our emergency room (ER). He had also received a living donor kidney transplantation 21 years ago from our institute. He had taken immunosuppressants twice a day regularly, which consisted of cyclosporin 50 mg twice a day and mycophenolate mofetil $720 \mathrm{mg}$ twice a day. Moreover, he had a history of Pneumocystis pneumonia (PCP) 4 years ago.

On neurological examination, he showed confused mentality and slow movement. He lost his left eyesight 13 years ago owing to retinal detachment of an unknown cause. Otherwise, a definitive focal neurologic deficit was not demonstrated. Routine laboratory tests, including infection markers, were within the normal range, and he had no fever.

A subsequent computed tomography (CT) scan showed 

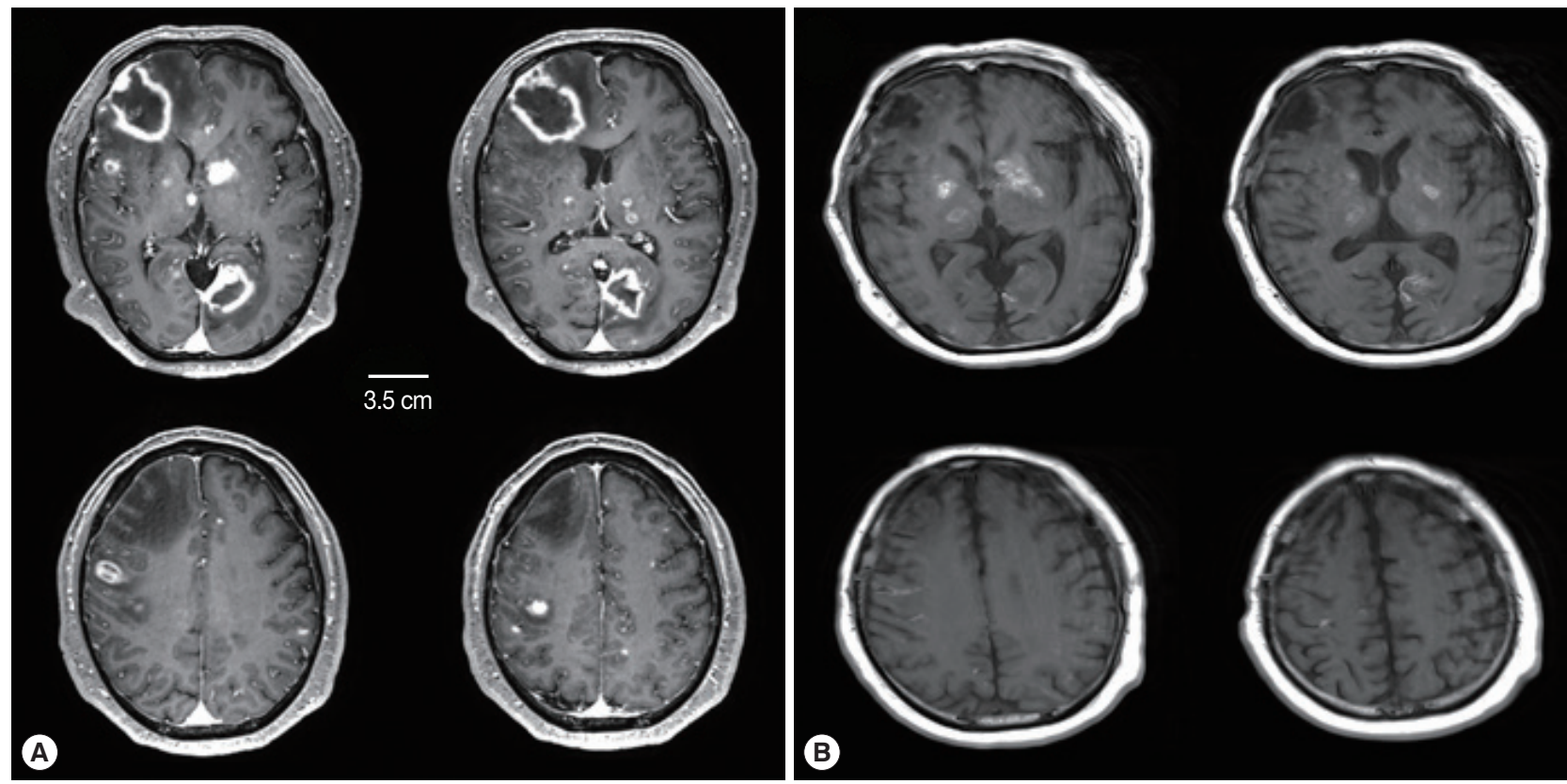

Fig. 1. Comparison of brain MRI between (A) at the ER visit and (B) 6 weeks after treatment. (A) Initial brain MRI showed multiple rim-enhancing lesions and miliary nodules. (B) Six weeks after treatment, rim-enhancing lesions disappeared with treatment-related changes.

multiple hypodense lesions mainly in the right frontal lobe and left occipital lobe. A magnetic resonance image (MRI) of the brain showed numerous additional miliary lesions, and multiple enhancing lesions on T1-weighted images were apparent in both thalami, the basal ganglia, and cortical and subcortical areas (Fig. 1A). The largest mass was a rim-enhancing $3.3 \times 4.6 \times 3.5 \mathrm{~cm}$ mass with perilesional edema in the right frontal lobe.

Considering his immune status, consultation with the infectious department for evaluating opportunistic infection of the brain proceeded. A serological test for toxoplasmosis was recommended.

Since the mental status of the patient had rapidly deteriorated to stupor at Day 3 of hospitalization, brain open biopsy was conducted to reduce the mass burden and for tissue confirmation by targeting the largest right frontal lobe mass. The mass was necrotic and had an extremely hard consistency. At this time, the serological results of toxoplasmosis were not yet available.

The examination of the frozen section was suggestive of malignant lymphoma versus inflammation. We waited for the final pathology results and administered steroids for 5 days. The report concluded that the lesion was cerebral toxoplasmosis (Fig. 2). High immunoglobulin G (317.6 IU/ml) and A (41.2 S/CO) from serologic tests for Toxoplasma gondii antibodies
(Detection kit: The Access Toxo IgG and IgM EIA systems) supported this conclusion. Additionally, polymerase chain reaction (PCR) results of the biopsied mass, which were reported 2 weeks after the surgery, confirmed cerebral toxoplasmosis (Fig. 3).

An initial regimen of pyrimethamine-sulfadiazine was used for 2 weeks. After the first regimen, considering the decrease in his kidney function, the combination of clindamycin and Bactrim (trimethoprim and sulfamethoxazole) was used until he died due to sepsis and recurrent gastrointestinal bleeding. During the treatment, his mental status waxed and waned but gradually decreased to stupor. Although brain MRI showed an improving state of cerebral toxoplasmosis lesions, his mental status did not significantly improve (Fig. 1B).

\section{DISCUSSION}

Late cerebral toxoplasmosis infection in patients with a kidney transplant has been rare. A literature review of 20 cases [2] showed that only 2 cases occurred more than 5 years after kidney transplantation. Seventeen cases occurred within 3 months. To the best of our knowledge, no case of cerebral toxoplasmosis occurring 20 years after kidney transplantation has been reported. There is only one case report in which cerebral toxoplasmosis occurred 15 years after kidney transplantation and 

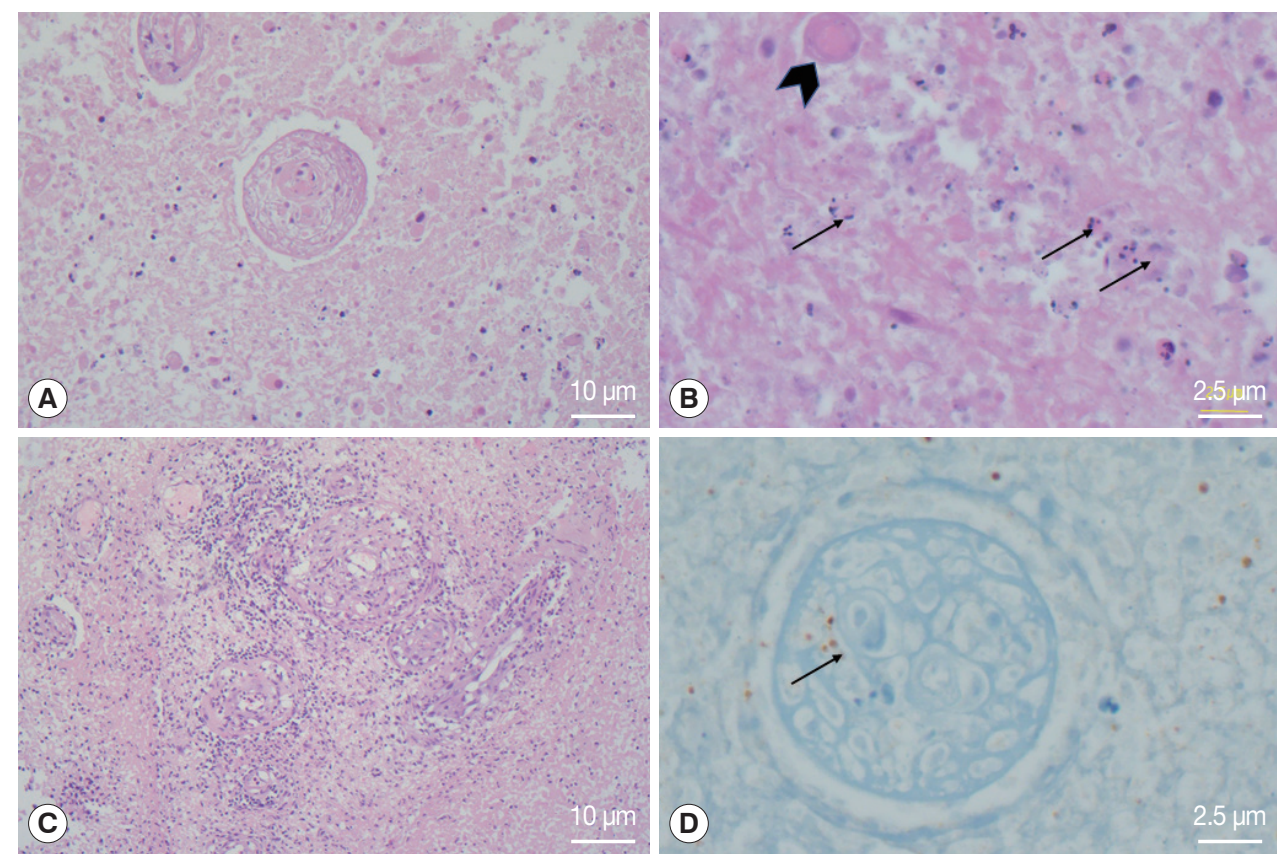

Fig. 2. Histological photomicrographs. (A) the lesion showed extensive coagulative necrosis (Hematoxylin Eosin $(\mathrm{HE}) \times 100)$. (B) Several tiny dot or crescent-like tachyzoites (arrows) and degenerated cysts-containing bradyzoites were found in the necrotic tissue (HE $\times 400)$. (C) Obliterative vasculitis with lymphoid cuffing was prominent in the peripheral part of the abscess (HEx 100). (D) Immunohistochemistry for Toxoplasma gondii showed positive reaction in the organisms inside the blood vessels and surrounding tissue $(\times 400)$.

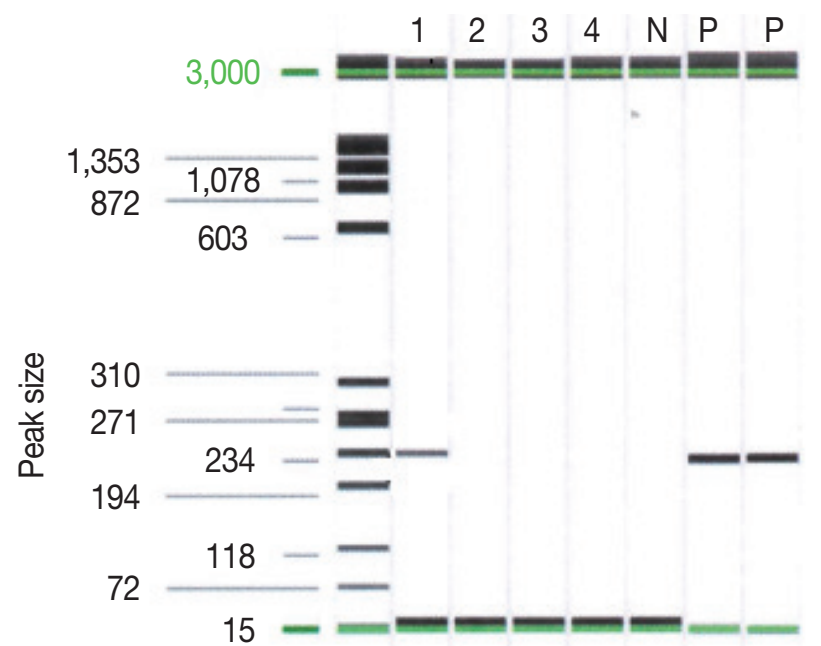

Fig. 3. PCR result on Toxoplasma gondii P30(SAG1) gene. Lane 1 indicated the sample of the patient. Lane $P$ indicated the positive control.

presented a course of progression similar to that of our patient [6]. Considering the lengthy period post kidney transplant, it was difficult to assume opportunistic infection such as toxoplasmosis as a primary diagnosis in this patient.

Additionally, multiple ring-enhancing lesions in the brain can occur across a diverse range of infections, neoplasms, or inflammatory and demyelinating causes [7]. Hence, several differential diagnoses need to be excluded first. A previous study showed that 221 rim-enhancing lesions were divided into gliomas (40\%), metastases (30\%), abscesses (8\%), and demyelinating disease $(6 \%)$ [8]. However, a patient's immune status should be checked before any differential diagnoses. In an immunocompromised host, it is known that the primary diagnosis of rim-enhancing lesions in the brain should include primary CNS lymphoma and toxoplasmosis [8].

In our patient, considering the infection history of the PCP and prolonged use of immunosuppressants, although it had been a long time, the possibility of opportunistic infection should have been more actively considered. However, assuming an exact diagnosis was time-consuming due to the unfamiliarity of the work-up process for CNS opportunistic infections. Additionally, serological tests for toxoplasmosis antibodies are not a routine test, and it took a long time to obtain the results.

The mortality rate of kidney transplant patients infected with toxoplasmosis has been reported at $64.5 \%$ [9]. Our patient died regardless of being on medication for toxoplasmosis within 2 weeks of his initial visit to ER. Since his mental status did not recover during the treatment period, he had been bed 
ridden, which caused associated complications that contributed to his death.

In Korea, because problems with toxoplasmosis infection are rare, routine serological tests do not include toxoplasmosis serology before kidney transplantation. In addition, there was no routine check-up protocol for brain imaging during the followup period. Our patient had no previous data on toxoplasmosis serology and had not undergone brain imaging despite being a patient at our hospital for 21 years after transplantation.

It is difficult to assume the exact time of the cerebral toxoplasmosis infection. Until his visit to the ER, a gradual progression of cerebral toxoplasmosis was clear based on the different stages of mass formation shown in the brain MRI.

His chief complaint leading to the ER visit might have been caused by lesions of the basal ganglia and thalamus. This might have been the time of the proliferative phase of these lesions because his mental status rapidly deteriorated while waiting for the pathology report.

Based on this tragic ending of our patient, early detection and early treatment of toxoplasmosis infection cannot be emphasized enough. From the moment he came to the ER, his fate might have been fixed. If he had received regular checkups of the brain during the follow-up period, his fate could have been changed. The current medical system could not conduct a rapid work-up process of toxoplasmosis infection. There is bound to be a delay. Even without definitive neurologic signs, regular inspection of brain imaging should be considered in these long-term immunocompromised patients.

\section{ACKNOWLEDGMENT}

This report was approved by our Institutional Review Board, and the requirement for informed consent was waived (IRB number: 20-2021-94).

\section{CONFLICT OF INTEREST}

The authors have no potential conflicts of interest to disclose.

\section{REFERENCES}

1. Saadatnia G, Golkar M. A review on human toxoplasmosis. Scand J Infect Dis 2012; 44: 805-814. https://doi.org/10.3109/00 365548.2012.693197

2. Da Cunha S, Ferreira E, Ramos I, Martins R, Freitas LD, Borges JL, Côrte-Real R, Mota A, Meliço-Silvestre A, Furtado AL. Cerebral toxoplasmosis after renal transplantation. Case report and review. Acta Med Port 1994; 7: 61-66. https://doi.org/10.20344/amp.3026

3. Hill D, Dubey JP. Toxoplasma gondii: transmission, diagnosis and prevention. Clin Microbiol Infect 2002; 8: 634-640. https://doi org/10.1046/j.1469-0691.2002.00485.x

4. Khurana S, Batra N. Toxoplasmosis in organ transplant recipients: evaluation, implication, and prevention. Trop Parasitol 2016; 6: 123-128. https://doi.org/10.4103/2229-5070.190814

5. Clissold R, Bingham C. Late presentation of toxoplasmosis in renal transplant recipients. NDT Plus 2010; 3: 480-482. https:// doi.org/10.1093/ndtplus/sfq113

6. de Joode AAE, Riezebos-Brilman A, Manson WL, Homan van der Heide JJ. Tissue is the issue: a solitary cerebral lesion 15 years after kidney transplantation. NDT Plus 2011; 4: 410-412. https:// doi.org/10.1093/ndtplus/sfr139

7. Garg RK, Sinha MK. Multiple ring-enhancing lesions of the brain. J Postgrad Med 2010; 56: 307-316. https://doi.org/10.4103/00223859.70939

8. Schwartz KM, Erickson BJ, Lucchinetti C. Pattern of T2 hypointensity associated with ring-enhancing brain lesions can help to differentiate pathology. Neuroradiology 2006; 48: 143-149. https:// doi.org/10.1007/s00234-005-0024-5

9. Renoult E, Georges E, Biava MF, Hulin C, Frimat L, Hestin D, Kessler M. Toxoplasmosis in kidney transplant recipients: report of six cases and review. Clin Infect Dis 1997; 24: 625-634. https://doi. org/10.1093/dind/24.4.625 